\title{
Numerical Simulation and Optimization for Double Pore Gas Drainage in Low-Permeability Fissured Coal Based on Lattice Boltzmann Method
}

\author{
Ming Gao \\ Key Lab Mine Disaster Prevention and \\ Control, Shandong University of \\ Science and Technology \\ College of Mechanical and Electronic \\ Engineering, Shandong Agricultural \\ University \\ Qingdao, China \\ lxgaom@163.com \\ Bin Fang \\ The First Area of Oxtgen \\ Procuction,Energy Factory of Benxi \\ Steel Plates Corp. Ltd \\ Benxi, China \\ sunboy0300@sina.com
}

\author{
Zhijie Wen* \\ Key Lab Mine Disaster Prevention and \\ Control, Shandong University of \\ Science and Technology \\ College of Mining and Safety \\ Engineering, Shandong University of \\ Science and Technology \\ Qingdao, China \\ sdust0532@gmail.com \\ Tao Yang \\ Faculty of Safety Engineering, North \\ China Institute of Science and \\ Technology \\ Yanjiao, China \\ Yangtao585@163.com
}

\author{
Xiaotong Li \\ Key Lab Mine Disaster Prevention and \\ Control, Shandong University of \\ Science and Technology \\ College of Mining and Safety \\ Engineering, Shandong University of \\ Science and Technology \\ Qingdao, China \\ lxt8588@outlook.com \\ Yujun Zuo \\ Mining School, Guizhou University \\ Guiyang, China \\ Zuo.yujun@yahoo.com.cn
}

Corresponding author: Zhijie Wen , sdust0532@gmail.com

\begin{abstract}
In order to simulate gas drainage in lowpermeability fissured coal considering double pores arranged symmetrically or asymmetrically, a dynamic model which can use a large pressure gradient is constructed based on Lattice Boltzmann Method (LBM). Different two pores drainage messures are studied respectively, including the conditions of the pores positions are asymmetrical or asymmetrical and the conditions of two pores depths are the same or not . Through analysis of the distributions of gas pressure and gas seepage flow in fissured coal at the steady gas seepage the reseach shows that:(1) With the increment of the drainage double pores depths arranged symmetrically or asymmetrically, the gas pressure reduce and the gas velocity qukien when the arrangement double pores asymmetrically with the different depths.(2) The deviation of the two pores arrangement to the centre in gas seepage field is large to be not recommanded attributing to the asymmetrical arrangement with large pores depths.(3)Under the condition of arrangement double pores symmetrically with the different depths, there is also a peak gas pressure in the gas seepage fields to be not effectively.(4)The gas drainage effect is the best when the pores are symmetrically arranged with the same depth,and with the increase of the number of pores, the drainage effect is better to be recommended.
\end{abstract}

Keywords-gas drainage, double pores, lattice boltzmann method, simulation

\section{INTRODUCTION}

Gas outburst disaster is one of the deadliest coal mine safety accidents. Gas outburst refers to the burst of coal and gas under certain conditions during coal mining. This kind of ejection is in shorter time dozens of seconds to several minutes and produce very huge impact force, destroy working face coal wall, discharge a lot of coal and gas from the depth of coal seam. The theory of gas seepage is a special theory to study the distribution of gas pressure and the law of flow change in coal. Due to the low permeability fracture coal and complex terrain conditions in China, underground gas extraction technology has become one of the important means of preventing and controlling gas outburst. As a result, significance has been attached with researches on both the gas seepage law and the optimal arrangement or depth of gas extraction pores in effective gas drainage and prevention of regional gas outburst.

Most mines are deep underground, the stress state of coal and rock is different with different depth and different geological conditions, and its permeability is affected by different degrees. Therefore, the difficulty and cost of experimental research and field testing technology are greatly increased. At the same time, the reliability of the results is questionable. Therefore, people need to find solutions to these problems. With the rapid development of computer science, people began to use computer to calculate the problems in science and engineering. After theoretical analysis method and scientific experimental research method, it gradually developed into another scientific research method.

A lot of works have been done on gas outburst mechanism, gas flow rules, gas extraction and drainage in low permeability fracture coal. Chinese scholars [1-5] and foreign scholars [6,7] studied the numerical simulation for gas seepage and drainage by finite element, finite difference, finite spectral method[8,9], and so on. Most of the seepage these models treat coal and gas as macroscopic media, and combine the classical mechanical method with the numerical simulation method to solve the problem. Although these methods are simple and convenient in calculation, they are not effective in reflecting the overall complexity of the system and the mutual movement of fluid particles. In 
computational fluid dynamics, the traditional numerical method is generally divided into two dimensions of macroscopic and microcosmic scale. Macroscopic analysis is based on continuity hypothesis by Navier - Stokes or Euler equation for the calculation and analysis of control equations. Which is a good way to solve some engineering on the macro scale of flow problems, but not all of the fluid flow problems can satisfy continuity hypothesis. While the microcosmic analysis is based on the molecular dynamics method to solve some microscopic scales fluid flow problem, but it is very difficult to calculate the irregular region boundary condition and it has higher requirements for computer hardware. Because the fracture coal body contains a large number of micro-and macro-scale pores at the same time, and its morphology is complex, it is difficult to accurately describe the flow of fluid in the traditional numerical simulation. Because the fracture coal body contains a large number of micro-and macro-scale pores at the same time, and its morphology is complex, it is difficult to accurately describe the flow of fluid in the traditional numerical methods, such as the finite element method.

In order to solve such problems, Lattice Boltzmann Method (LBM), which has been widely applied in recent years[10-15], is based on the molecular dynamics theory. LBM builds a discrete velocity model on the micro scale to simulate the movement of the fluid particles, and obtains the macroscopic motion variables of the fluid through statistical methods. This method can be regarded as a simplified model of molecular dynamics, which adopts the method of nonequilibrium statistical physics to study the motion law of fluid with the classical Boltzmann equation. LBM not only retains various advantages of the LGA method, but also overcomes its own disadvantages, making it a very suitable method in seepage simulation.

In recent years, Tan [16,17] and his coauthors Teng[18-19] and Gao[20-22] take the lead to establish the gas seepage calculation model by LBM, analyzed the Klinkenberg effect and adsorption - desorption characteristics of seepage, the effects of gas flow in coal is studied and the evolution of the pressure distribution in time and space distribution rule, analyzed the influence of different crack distribution on the gas flow in coal, the pressure of drainage and drainage holes layout on the effects of the gas drainage and coal mesoscopic structure of image processing and the combination of LBM, preliminary study the real seepage law of gas in coal body.

In the case of double pores gas drainage in the fissured coal, $\mathrm{Li}$ and Gao [21,22] analyzed the effects of the pores depth and separation distances arranging the double pores symmetrically on the gas seepage flow field. While arranging the double drainage pores symmetrically or asymmetrically may bring the changes of gas pressure and gas seepage flow. When the gas seepage pressure peak is very high the gas burst may occur. Reasonable arrangement the double pores and their depth are very crucial and of theoretical and practical significance. In this paper , firstly we use the model of double gas drainage in fissured coal [21$22]$ to analyze the effect of pore depth when the double pore asymmetrically. After that, off-center double pores with same depth conditions is obtained. Thirdly the double pores arranged symmetrically with different depths are discussed. Finally, the best drainage method of arranging the double pores symmetrically with the same depth is recommended.

\section{LATTICE BOLTZMANN EQUATION}

As a molecular dynamic simulation model, LBM Seepage equation standard form can be written as LBM equation[20,21,23-27]:

$$
g_{i}\left(x+c_{i} \Delta t, t+\Delta t\right)-g_{i}(x, t)=-\frac{1}{\tau}\left[g_{i}(x, t)-g_{i}^{\text {eq }}(x, t)\right]+\Delta t F_{i}
$$

where $\tau=\tau_{0} / \Delta t$ is the dimensionless relaxation time, $\Delta t$ is the time increment, $g_{i}(x, t)$ is the pressure distribution function for the particle with velocity $\boldsymbol{c}_{i}$ at position $\boldsymbol{x}$ and time $t, g_{i}^{e q}(x, t)$ is the Equilibrium Distribution Function (EDF).

For the EDF in D2Q9 model, the most extensive used model, is defined as

$$
g_{i}^{e q}=\omega_{i}\left[\frac{\phi p}{c_{s}^{2} \rho}+\frac{\boldsymbol{c} \cdot \boldsymbol{u}}{c_{s}^{2}}+\frac{\boldsymbol{u} \boldsymbol{u}:\left(\boldsymbol{c}_{i} \boldsymbol{c}_{i}-c_{s}^{2} \boldsymbol{I}\right)}{2 \phi c_{s}^{4}}\right]
$$

where $\omega_{i}$ is the weight, $C_{s}$ is the sound velocity, In extensive usemodel D2Q9[20], the discrete velocities are givenby $\boldsymbol{c}_{0}=0, \boldsymbol{c}_{1}=-\boldsymbol{c}_{3}=c(1,0), \boldsymbol{c}_{2}=-\boldsymbol{c}_{4}=c(0,1)$, $\boldsymbol{c}_{5}=-\boldsymbol{c}_{7}=c(1,1), \boldsymbol{c}_{6}=-\boldsymbol{c}_{8}=c(-1,1)$, the parameter $C_{s}$ is defined as $c_{s}^{2}=c^{2} / 3$.The weights are given by $\omega_{0}=4 / 9, \omega_{1 \sim 4}=1 / 9$, and $\omega_{5 \sim 8}=1 / 36$.the macroscopic pressure $p$ and velocity $\boldsymbol{u}$ are defined as

$$
\frac{c_{s}^{2} \rho}{\phi} \sum_{i} g_{i}=p, \boldsymbol{u}=\frac{\boldsymbol{v}}{c_{0}+\sqrt{c_{0}^{2}+c_{1}|\boldsymbol{v}|}}
$$

Where $\boldsymbol{v}$ is given by

$$
\boldsymbol{v}=\sum_{i} \boldsymbol{c}_{i} f_{i}+\frac{\delta_{t}}{2} \phi \boldsymbol{G}
$$

Then the macroscopic equations deduced as (See reference $[20,21,27]$ for details):

$$
\begin{gathered}
\frac{1}{c_{s}^{2}} \partial_{t}(\phi p)+\nabla \cdot \boldsymbol{u}=0 \\
\partial_{t} \boldsymbol{u}+\boldsymbol{u} \cdot \nabla\left(\frac{\boldsymbol{u}}{\phi}\right)=-\frac{1}{\rho} \nabla(\phi p)+v_{e} \nabla^{2} \boldsymbol{u}+\boldsymbol{F}
\end{gathered}
$$

Where $v_{e}=C_{s}^{2}(\tau-0.5) \Delta t$ is an effective viscosity coefficient.

Ignoring the moving accelerate term $\boldsymbol{u} \cdot \nabla\left(\frac{\boldsymbol{u}}{\phi}\right)$ and the viscosity factor, (5.b) can be rewritten by

$$
\partial_{t} \boldsymbol{u}=-\frac{1}{\rho} \nabla(\phi p)
$$

\section{GAS FLOW FIELD MODEL}

Teng ,Tan and Gao etc.[16-22] used LBM to establish a seepage model and studied the flow and pressure distribution of gas in coal. The geometric model is shown in Figure 1. The model was divided into $80 \times 80$ grids, and dimensionless 
processing was used to calculate the size, pressure and velocity of the convection field. As the flow of gas in coal will be affected by a variety of conditions, the main factors are only taken into account for the convenience of calculation. The model is assumed as follows: The change of gas pressure in overburden coal has no effect on porosity. The temperature change of the gas flow field is ignored. The influence of gas volume force is not considered.

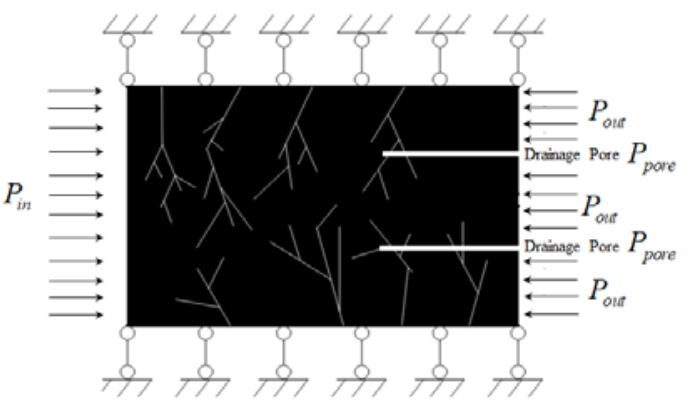

Fig.1. Gas flow field model

The drilling parameters that affect the effect of gas drainage are: pore diameter, hole Angle, pore length, pore spacing, pore number, negative pressure suction, hole sealing material and process. [20-22] in this paper, several factors affecting gas drainage effect are mainly discussed: (1) the pore depth(2) drainage arranged symmetrically or not(3) drainage symmetrically of double pores with different depth.

\section{ARRANGEMENT DOUBLE PORES ASYMMETRICALLY WITH THE DIFFERENT DEPTHS}

The Figure.2 Figure.3 and Figure.4 are gas seepage pressure diagram and velocity streamline diagram of different pore depth when the pore arrangement position is 3060 (drainage asymmetrically), the pressure on the left side is 1.01 , free seepage pressure on the right side is 1 , drainage pressure at the pore is 0.96 . Without loss of generality all of the pressures are dimensionless.

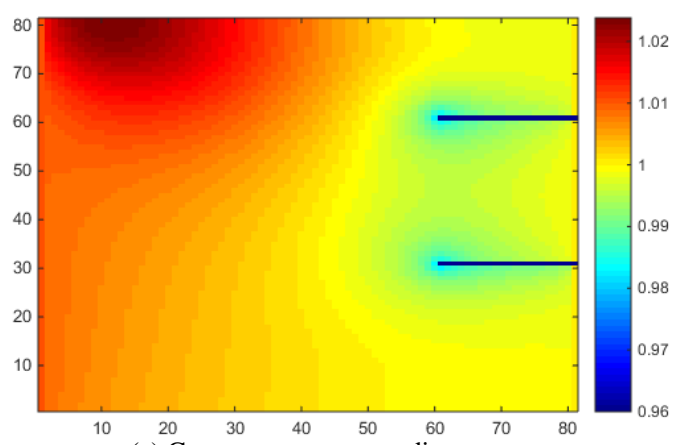

(a) Gas seepage pressure diagram

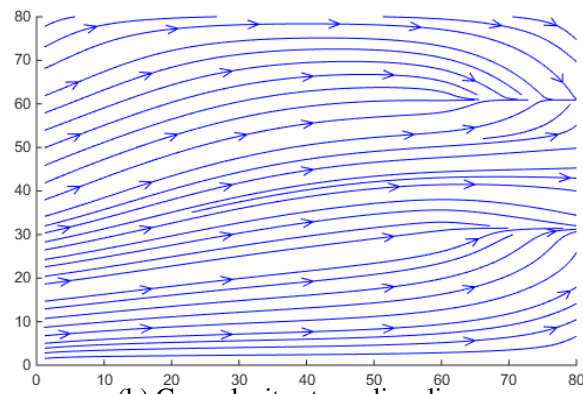

${ }^{10}$ (b) $\stackrel{20}{20} \stackrel{30}{ } \stackrel{40}{40} \stackrel{50}{50} \stackrel{60}{70}$

Fig.2.Gas seepage pressure diagram and velocity streamline diagram of 20 opening depth(opening position:30 60)

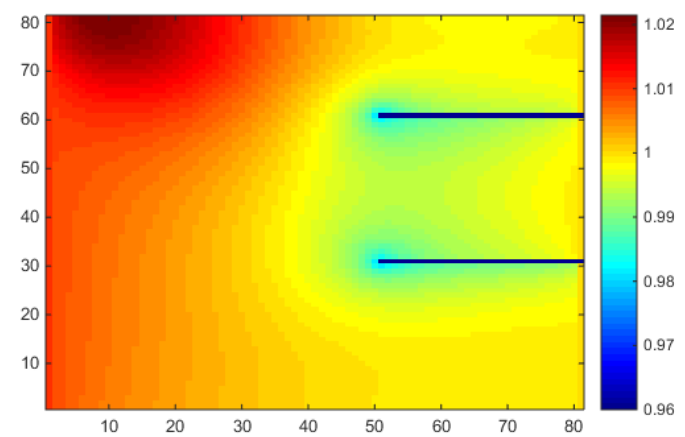

(a) Gas seepage pressure diagram

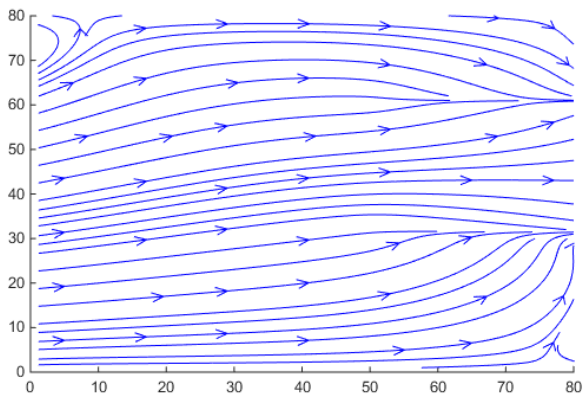

(b) Gas velocity streamline diagram

Fig.3.Gas seepage pressure diagram and velocity streamline diagram of 30 opening depth(opening position:30 60)

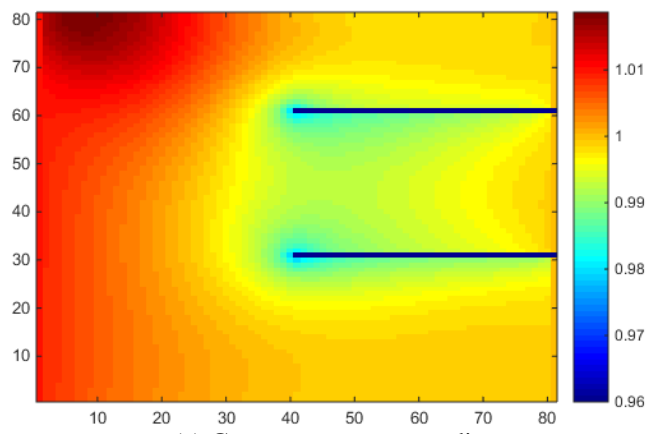

(a) Gas seepage pressure diagram

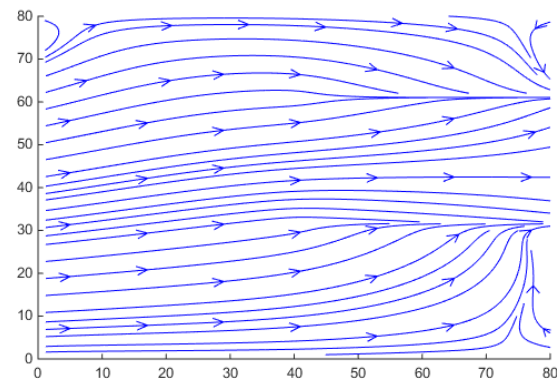

(b) Gas velocity Streamline diagram

Fig.4.Gas seepage pressure diagram and velocity streamline diagram of 40 opening depth(opening position:30 60)

As can be seen from the above figures, the pressure in the upper half of the figure decreases in sequence from the left boundary to the pore opening stage, and the pressure at the opening part in linear distribution varies greatly. With the deepening of the pore opening depth, the pressure gradient in the flow field becomes larger, the pressure change obviously, and the region where the lower left pressure increases decreases,this may occure gas burst.

The flow velocity of gas at the pore opening is large, and also near the edge of the opening, the deeper the opening, the 
closer the gas flow is to the radial flow, while in the far away region, the laminar flow, the direction of gas flow near the opening changes faster. With the increase of the opening depth, the gas flow in the upper right and the lower right boundary successively forms vortex, the vortex in the upper left gradually decreases, and the gas trace in the flow field becomes more and more dense, indicating that the gas velocity becomes larger.

To sum up, the deeper the opening depth, the greater the pressure change, the denser the gas flow trace, the better the drainage effect.

\section{ARRANGEMENT DOUBLE PORES ASYMMETRICALLY WITH THE SAME DEPTH}

Figure 5 and Figure 6 are gas seepage pressure diagram and velocity streamline diagram of 1060 pore position and 2070 pore position when the pore opening position is 3060 respectively,the pressure on the left side is 1.01 , free seepage pressure on the right side is 1 , drainage pressure at the pore is 0.96. All of the pressures are also dimensionless .

Comparing Figure 5 and Figure 6, it can be seen that both of the two gas pressure and gas flow are symmetrical. It follows that: Asymmetric pore openings on both sides of the right boundary center, when the distance between the double pores is equal, drainage effect is the same.

There is another important point can be obained from the Figure 4 and Figure 5, that the further of pores distance is the worse of the drainage the effect is.which attributes to the gas pressure distribution near the drainage pores.

As a result, dranaige asymmetrically is not recommanded while insite of enlarging the pores depth.

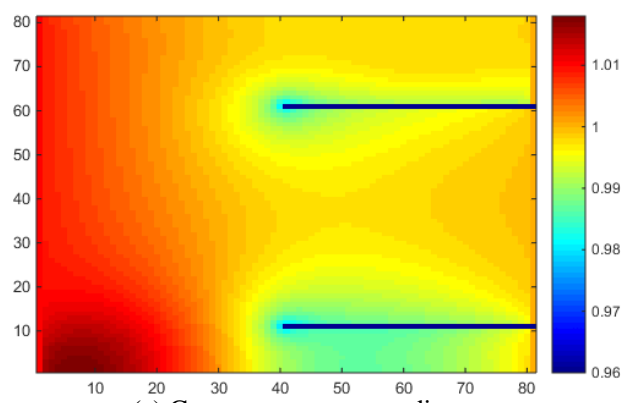

(a) Gas seepage pressure diagram

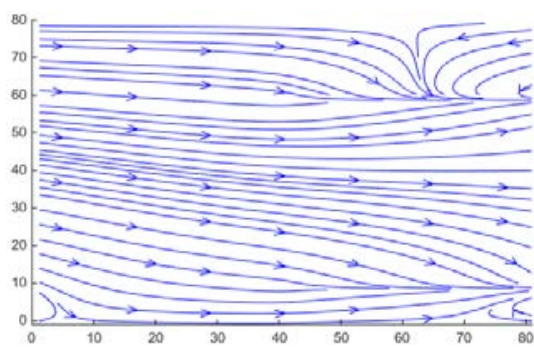

(b) Gas velocity streamline diagram

Fig.5.Gas seepage pressure diagram and velocity streamline diagram of 10 60(pore depth:40)

\section{ARRANGEMENT DOUBLE PORES SYMMETRICALLY WITH DIFFERENT DEPTHS}

Figure 7 and Figure 8 are the gas seepage pressure diagram and the gas velocity streamline diagram when the double pores depth are 3050 and 5030 respectively, the arrangment of opening pores position is symmetrical.

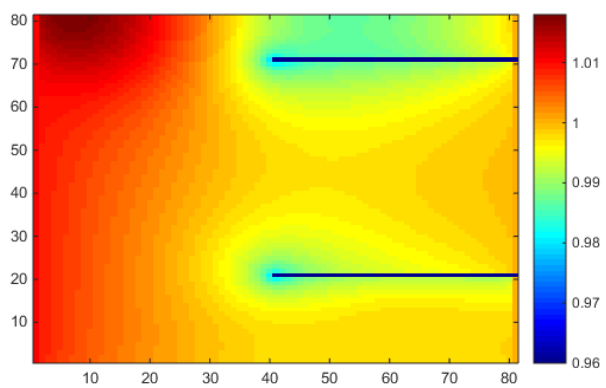

(a) Gas seepage pressure diagram

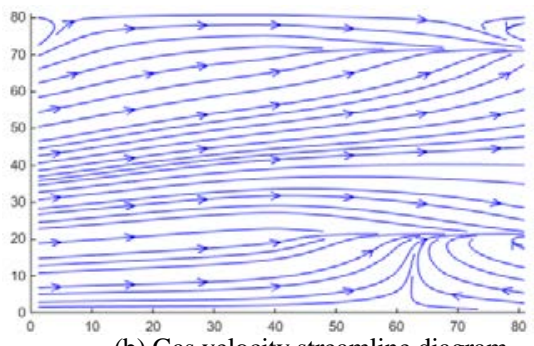

(b) Gas velocity streamline diagram

Fig.6.Gas seepage pressure diagram and velocity streamline diagram of 20 70(pore depth:40)

The distributions rules of gas pressure and seepage low of (a)(b) in Figure 7 and (a) (b) in Figure 8 are symmetrical respectively. In both cases, there was a significant pressure surge and a significant vortex on one side of the deeper pore.

The pores position and pores depths in the two conditions are symmetrical with the same sum of depth, so that the pressure distribution and velocity streamline of the model are symmetrical, which shows that the drainage effect is the same in both cases, and the drainage effect is not good.

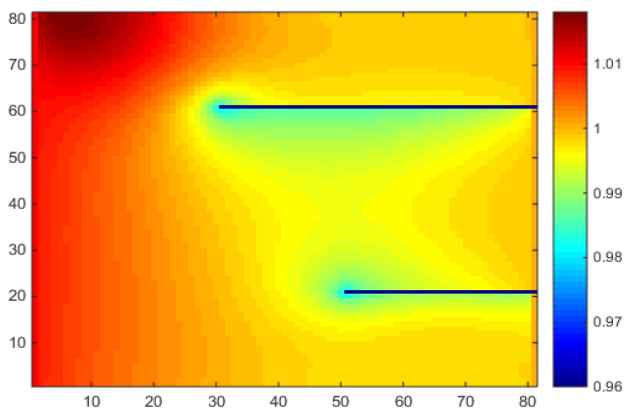

(a) Gas seepage pressure diagram

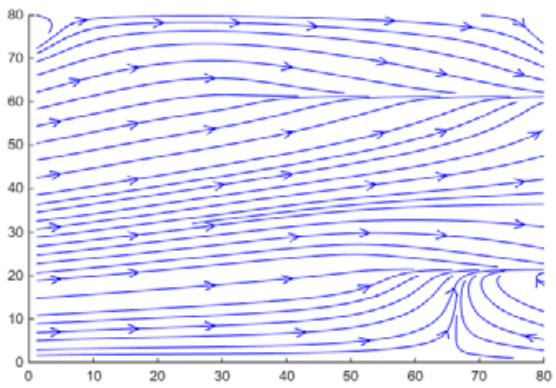

(b) Gas velocity streamline diagram

Fig.7.Gas seepage pressure diagram and velocity streamline diagram of 3050 opening depth(opening position:20 60) 


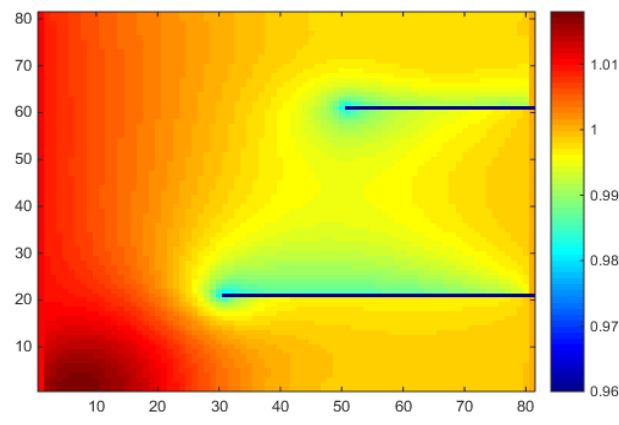

(a) Gas seepage pressure diagram

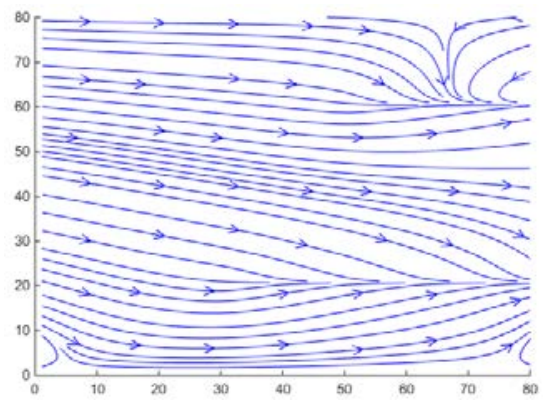

(b) Gas velocity streamline diagram

Fig.8.Gas seepage pressure diagram and velocity Streamline diagram of 50,30 opening depth(opening position:20,60)

While near the position where the deeper of two pores, there is a peak of gas pressure where the vortex occurs. Hence this drainage condition is not recommended that the two pores arrangmenr are symmetical although the two pores depths are different.

\section{ARRANGEMENT PORES SYMMETRICALLY WITH THE SAME DEPTH}

The above analysis of gas pressure field has a region of great pressure, gas flow trace local concentration, which is easy to cause gas outburst. The figure below shows gas seepage pressure and the gas velocity streamline when both the pore position and double pores are all symmetrical in gas seepage field.

Compared with the previous three cases, it is not difficult to find that when the opening positions of two pores are symmetrical, the pressure change in the flow field is larger, and the pressure gradient from the gas entering the flow field to the orifice is larger, which is beneficial to the flow of gas in the flow field, and the flow line diagram in the middle of the flow field is denser and the gas velocity is faster. Therefore, the gas drainage effect of symmetrically opening double pores is good and effectively.

When the number of pores are increased from two to three, the pressure of gas in the gas flow field changes more, as illustrats in Figure 10, the pressure on the right side of the figure decreases. Compared with the double pores, the vortex phenomenon on the right side disappears when the three pores are opened, and the streamlines in the flow field are more dense.

With the increase of the number of openings, the pressure at the right opening becomes lower and the gas velocity in the flow field increases, which is beneficial to gas drainage.

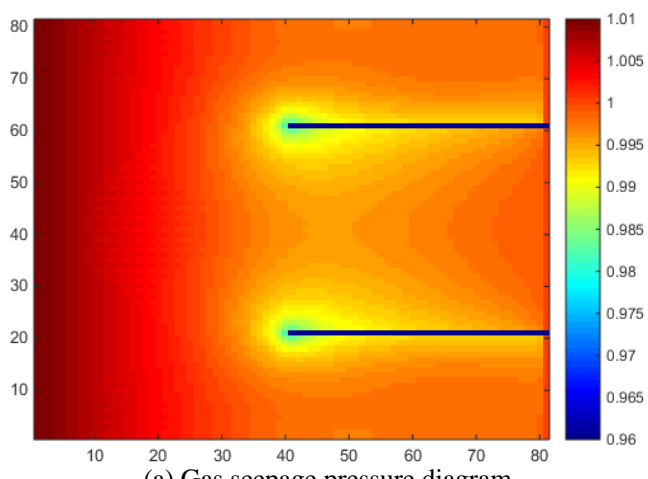

(a) Gas seepage pressure diagram

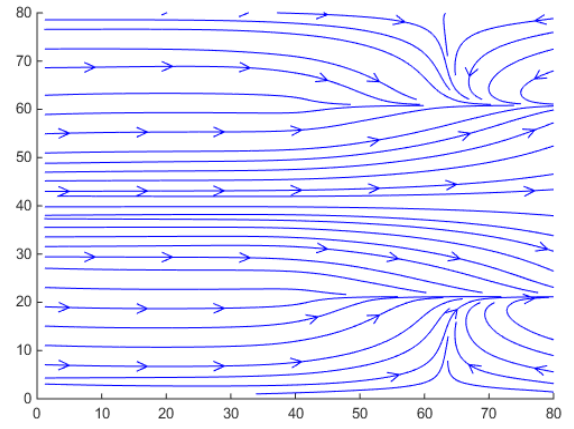

(b) Gas velocity streamline diagram

Fig.9.Gas seepage pressure diagram and velocity Streamline diagram of central symmetric double pores(opening position:20 60,depth:40)

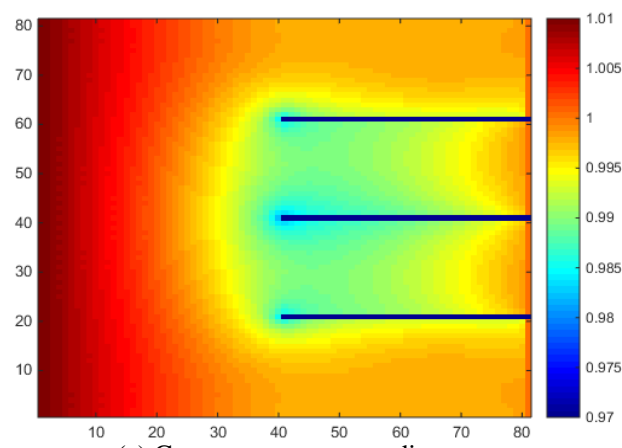

(a) Gas seepage pressure diagram

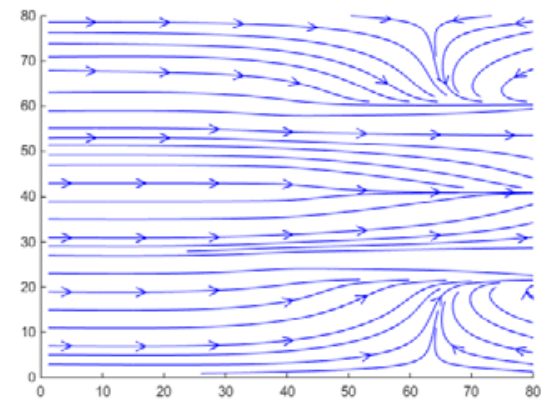

(b) Gas velocity streamline diagram

Fig.10.Gas seepage pressure diagram and velocity Streamline diagram of central symmetric three pores(opening position:20 40 60,depth:40)

\section{CONCLUSION}

In this paper, the LBM is used to simulate gas drainage in the fissured coal arranging the double pore symmetrical or asymmetrical. Through simulating the gas drainage under the conditions of the pores positions are asymmetrical or asymmetrical and the conditions of two pores depths are the same or not, the gas distributions of gas pressure and gas 
seepage flow are analyzed respectively. The following conclusions are obtained:

(1)The deeper the pore opening depth is, the greater the pressure change higher, the denser the gas flow trace is and the better drainage effect is, when the arrangement double pores asymmetrically with the different depths.

(2)When the double pores positions is asymmetrical with the depths changes, drainage asymmetrically is not recommended. While the deviation of the two pores arrangement to the center in gas seepage field is large to be not recommended attributing to the asymmetrical arrangement.

(3)Under the condition of arrangement double pores symmetrically with the different depths, the drainage effect is better than above conditions.

(4)The gas drainage effect is the best when the pores are symmetrically arranged with the same depth. With the increase of the number of openings, the gas pressure becomes lower and the gas velocity in the flow field increases.

\section{ACKNOWLEDGMENT}

In this paper, the research was sponsored by the State Key Research Development Program of China (Grant No.2016YFC0600708), the National Natural Science Foundation of China (Grant No. 51605264), Tai'shan Scholar Engineering Construction Fund of the Shandong Province of China; Tai'shan Scholar Talent Team Support Plan for Advanced \& Unique Discipline Areas. Shandong Province Higher Educational Science and Technology Program(Grant No.J15LH04), the Natural Science Foundation of Shandong Province (Grant No.ZR2018 MEE001, \& No. ZR2018BA022), State Key Laboratory of open funds(No. MDPC201601)

\section{REFERENCES}

[1] Zhou Shi-ning, "Mechanism of gas flow in coal," Journal of China coal society, vol.15, pp. 61-67,1990.

[2] PAN Li-you, HUANG Shou-qing, CHEN Li-qiang, KONG Fan-peng, "Establishment and application of the stereo drainage model for high gas working face of short distance seam group,” Journal of China Coal Society, vol. 37, pp. 1461-1465, 2012,

[3] GUO Peihong, LI Haixia, ZHU Jianan, "Numerical simulation of gas drainage from boreholes in coal seams," Journal of Liaoning Technical University, vol.28, pp. 260-262, 2009.

[4] HAO Fu-chang, LIU Ming-ju, SUN Li-juan, "Determination method of gas drainage radius based on multi-physics coupling," Journal of China Coal Society, vol. 38, pp. 106-111, 2013.

[5] ZHOU Fu-bao, LI Jin-hai, ZE Xi, LIU Ying-ke, ZHANG Ren-gui, SHEN Si-jun, "A Study of the second hole sealing method to improve gas drainage in coal seams,” Journal of China University of Mining\& Technology, vol. 38, pp. 764-768, 2009.

[6] Taheri Adel, Sereshki Farhang, Ardejani Faramarz Doulati, Mirzaghorbanali Ali, "Simulation of macerals effects on methane emission during gas drainage in coal mines,”. Fuel, vol. 210, pp. 659665, 2017.

[7] Danesh Nima N., Chen Zhongwei, Aminossadati Saiied M, "Impact of creep on the evolution of coal permeability and gas drainage performance,” Journal of Natural Gas Science and Engineering, vol. 33, pp.469-482, 2016.
[8] C.Yu, X.Xian, "Analysis of gas seepage flow in coal beds with finite element method," Symposium of 7th international conference of FEM in flow problems, Huntsvill, USA, 1989.

[9] C.Yu, X.Xian, "A boundary element method for inhomogeneous medium problems,” Proceedings: 2nd world congs.On computational mechanics, Stuttgart, FRG, 1990.

[10] Guo Z-L, Shi B-C, Wang N-C, "Lattice BGK model for incompressible Navier-Stokes equation,” Journal of Computational Physics, vol. 165, pp.288-306, 2000.

[11] Tan Wei, Wu Hao, Zhu Guorui, "Fluid-structure interaction using lattice Boltzmann method: Moving boundary treatment and discussion of compressible effect,”. Chemical Engineering science, vol. 184, pp.273-284, 2018

[12] Hajabdollahi Farzaneh, Premnath Kannan N, “Central moments-based cascaded lattice Boltzmann method for thermal convective flows in three-dimensions,” International Journal of Heat and Mass Transfer, vol. 120, pp.838-850, 2018.

[13] Sommerfeld M., Qadir Z., "Fluid dynamic forces acting on irregular shaped particles: Simulations by the Lattice-Boltzmann method," International Journal of Multiphase Flow, vol.101, pp.212-222, 2018.

[14] Patel Ravi A., Perko Janez, Jacques Diederik, “A three-dimensional lattice Boltzmann method based reactive transport model to simulate changes in cement paste microstructure due to calcium leaching," Constuction and Building Materials, vol.166, pp.158-170, 2018.

[15] Zhuo Congshan, Sagaut Pierre, “Acoustic multipole sources for the regularized lattice Boltzmann method: Comparison with multiplerelaxation-time models in the inviscid limit," Physical Review E, vol.95. p.063301, 2017.

[16] Yun-Liang, T., T. Gui-Rong and Z. Ze, “A modified LBM model for simulating gas seepage in fissured coal considering Klinkenberg effects and adsorbability-desorbability," Chinese Physics Letters, vol.27, p. 014701, 2010.

[17] Tan Yunliang, Yin Yanchun, Teng Guirong, etc., "Simulation research of gas seepage based on Lattice Boltzmann Method,” Journal of China Coal Society, vol. 39, pp.1446-1454, 2014.

[18] Guirong Teng, Yunliang Tan, Ming Gao and Zhigang Zhao, "Simulation of gas drainage in fissured coal based on Lattice Boltzmann Method,” Journal of China Coal Society, vol. 33, pp.914919, 2008.

[19] Teng Guirong, Tan Yunliang, Gao Ming, "Simulation of gas seepage in fissured caol based on Lattice Boltzmann Method," Chinese Journal of Rock Mechanics and Engineering, vol. 26, pp.3503-3508, 2007.

[20] Gao Ming, Tan Yun-liang, Teng Gui-rong, "Numerical Simulation of Gas Drainage in Low-Permeability Fissured Coal,” 2008 International Workshop on Modelling, Simulation and Optimization, Hong Kong, pp. 384-387, 2008.

[21] Xiaotong Li, Kun Wang, Ming Gao, "Simulations on two holes drainage for cleat coal gas," IOP Conf. Series: Earth and Environmental Science, vol. 170 , p. 022029, 2018.

[22] Xiaotong Li, Ming Gao, "Influence factors of two holes drainage for cleat coal gas,” IOP Conf. Series: Earth and Environmental Science, vol. 170 , p. $022054,2018$.

[23] Peng Cheng, Geneva Nicholas, Guo Zhaoli, "Direct numerical simulation of turbulent pipe flow using the lattice Boltzmann method,” Journal of Computational Physics, vol.357, pp.16-42, 2018.

[24] Hu Junjie, Tao Shi, Guo Zhaoli, “An efficient unified iterative scheme for moving boundaries in lattice Boltzmann method," Computers \& Fluids, vol.144, pp.31-43, 2017.

[25] Peng Cheng, Geneva Nicholas, Guo Zhaoli, “Issues associated with Galilean invariance on a moving solid boundary in the lattice Boltzmann method,” Physical Review E, vol. 95, p.013301, 2017.

[26] Meng Xuhui, Guo Zhaoli, "Boundary scheme for linear heterogeneous surface reactions in the lattice Boltzmann method," Physcial Review E, vol. 94, p.053307, 2017.

[27] Zhaoli Guo. Lattice Boltzmann Method for Hydro dynamics, Science Press, Hubei China, 2002. 